\title{
Impact of adolescent sucrose access on cognitive control, recognition memory, and parvalbumin immunoreactivity
}

\author{
Amy C Reichelt, ${ }^{1}$ Simon Killcross, ${ }^{1}$ Luke D. Hambly, ${ }^{1}$ Margaret J. Morris, ${ }^{2}$ and \\ R. Fred Westbrook ${ }^{1}$ \\ ${ }^{1}$ School of Psychology, ${ }^{2}$ School of Medical Science, University of New South Wales Australia, Kensington, Sydney, New South Wales \\ 2052, Australia
}

\begin{abstract}
In this study we sought to determine the effect of daily sucrose consumption in young rats on their subsequent performance in tasks that involve the prefrontal cortex and hippocampus. High levels of sugar consumption have been associated with the development of obesity, however less is known about how sugar consumption influences behavioral control and high-order cognitive processes. Of particular concern is the fact that sugar intake is greatest in adolescence, an important neurodevelopmental period. We provided sucrose to rats when they were progressing through puberty and adolescence. Cognitive performance was assessed in adulthood on a task related to executive function, a rodent analog of the Stroop task. We found that sucrose-exposed rats failed to show context-appropriate responding during incongruent stimulus compounds presented at test, indicative of impairments in prefrontal cortex function. Sucrose exposed rats also showed deficits in an on object-in-place recognition memory task, indicating that both prefrontal and hippocampal function was impaired. Analysis of brains showed a reduction in expression of parvalbumin-immunoreactive GABAergic interneurons in the hippocampus and prefrontal cortex, indicating that sucrose consumption during adolescence induced long-term pathology, potentially underpinning the cognitive deficits observed. These results suggest that consumption of high levels of sugarsweetened beverages by adolescents may also impair neurocognitive functions affecting decision-making and memory, potentially rendering them at risk for developing mental health disorders.
\end{abstract}

Excessive consumption of high sugar foods and drinks, which are both cheap and readily available, plays a central role in development of obesity in humans. Increasing sugar consumption affects the majority of the developed and developing world (Lustig et al. 2012; Basu et al. 2013) and a major contributor to this increase is the widespread availability of relatively cheap sugar-sweetened beverages or soft drinks (Guthrie and Morton 2000; Popkin and Nielsen 2003). Consumption of soft drinks has been shown to contribute to $80 \%$ of the increase in added sugar consumption during the period 1962-2000 in the U.S. (Popkin and Nielsen 2003) and today are the largest single source of added sugar consumption (Yang et al. 2014).

While the consequences of sugar overconsumption on physical health, including weight gain, diabetes, metabolic syndrome, and cardiovascular diseases are well known (Berkey et al. 2004; Dubois et al. 2007), less is known about how sugar affects cognition, behavior, and mental health. Adults consuming high levels of soft drinks $(>500 \mathrm{~mL} / \mathrm{d}$ ) had $\sim 60 \%$ greater risk of suffering from depression, stress-related problems, suicidal ideation, psychological distress, or indeed some current mental health condition than those not consuming soft drinks (Shi et al. 2010), and odds ratios for depressive symptoms were greater in adults consuming high levels of soft drinks (Guo et al. 2014; Yu et al. 2014). Of particular concern is the fact that sugar intake is higher among adolescents than in any other age group (Frary et al. 2004; Bremer and Lustig 2012). Moreover, odds ratios for measures of mental distress, hyperactivity, and conduct problems were highest in adolescents self-reporting the greatest levels of soft drink

\section{Corresponding author: a.reichelt@unsw.edu.au}

Article is online at http://www.learnmem.org/cgi/doi/10.1101/Im.038000.114. consumption ( $>800 \mathrm{~mL} / \mathrm{d}$ (Lien et al. 2006)). Furthermore, a recent study of adolescents used a Go/No Go task to measures response control and sustained attention, and found greater inhibitory problems in males, but not females, who consumed larger volumes of soft drinks (Ames et al. 2014). Rodent models have shown that animals who over-consumed sugar during adolescence, but not in adulthood, were less motivated to respond for sweet (saccharin) and nonsweet solutions (maltodextrin), but not cocaine self-administration as adults (Vendruscolo et al. 2010). This reduction in motivation, or anhedonia, is viewed as a central symptom in neuropsychiatric disorders including depression, schizophrenia, eating disorders, and drug addiction, raising the possibility that excessive sugar consumption in adolescence could be a risk factor for a core characteristic of such disorders.

Sugar is a potent natural reward, and allowing rats access to sugar over several weeks has been shown to produce neurobiological perturbations characteristic of addiction such as reduced dopamine D2 receptors in the striatum and increased D1 receptor binding in the nucleus accumbens (NAc) core and shell (Colantuoni et al. 2001). Brain reward systems including the mesocorticolimbic dopamine system mediate both the motivation to procure (wanting) and the hedonic impact (liking) of both psychoactive drugs and natural rewards such as palatable drinks

(C) 2015 Reichelt et al. This article is distributed exclusively by Cold Spring Harbor Laboratory Press for the first 12 months after the full-issue publication date (see http://learnmem.cshlp.org/site/misc/terms.xhtml). After 12 months, it is available under a Creative Commons License (AttributionNonCommercial 4.0 International), as described at http://creativecommons. org/licenses/by-nc/4.0/. 
and foods (Koob and Volkow 2010; Berridge 2012). However, separate neural mechanisms that underpin self-administration of food and drugs such as cocaine have been observed. Dopamine transporter knockout mice have low rates of cocaine self-administration, but no difference in responding for sucrose (Thomsen et al. 2009) and contrasting roles of subsets of NAc neurons have been observed when rats respond for natural or drug rewards (Cameron and Carelli 2012). Furthermore, chronic exposure to drugs of abuse is associated with deficits in cognitive function and behavioral control (Nelson and Killcross 2006; Parsegian et al. 2011; Porter et al. 2011). Thus, it is possible that consumption of diets high in sugar or exposure to psychoactive drugs during critical periods of brain development (e.g., adolescence) may produce enduring changes in the brain reward system and also in cognitive control (Smith 2003; Vendruscolo et al. 2008; Iniguez et al. 2009; Zamberletti et al. 2014). We sought to determine whether consumption of sweetened drinks earlier in life might also produce similar persistent changes in brain regions involved in high-order cognition in adulthood. We used a limited access schedule in which rats were provided access to sucrose for $2 \mathrm{~h}$ each day for $28 \mathrm{~d}$. This schedule has been shown to impair performance by adult rats in a task that relies upon frontostriatal function, specifically goal-directed responding that relies upon behavior (Kendig et al. 2013). Regions of particular interest are the prefrontal cortex (PFC), and the hippocampus, brain regions critical for decision-making and various forms of memory, respectively (Euston et al. 2012; Yu and Frank 2014).

The present experiment had three aims. The first was to examine whether limited daily access to a sucrose solution across adolescence exerted long-term effects on the use of contextual cues to disambiguate responding to stimulus compounds using a biconditional discrimination task. This behavioral assay models elements of response conflict seen in the Stroop task (Stroop 1935), a cognitive task that is often used to study interference and attention in humans and is highly sensitive to frontal lobe dysfunction (MacLeod 1991; MacLeod and MacDonald 2000; Stuss et al. 2001). The rodent analog of the Stroop task examines choice behavior under conditions of cue and response conflict, and depends on the integrity of the medial PFC (Haddon and Killcross 2006; Reichelt et al. 2013b). On the basis of anatomical criteria including granular cytoarchitecture and connectivity with the mediodorsal thalamus, there is general agreement that rodents have a frontal region that bears homologies to the primate PFC (e.g., Groenewegen 1988; Uylings et al. 2003). Functionally, the dorsolateral PFC and ventromedial PFC are thought to be integrated within rodent medial PFC (Uylings et al. 2003). Disruption of the rodent mPFC impairs the use of task-setting cues to control the selection of responses in conflict situations (Haddon and Killcross 2006, 2007; Marquis et al. 2007; Reichelt et al. 2013b).

The second aim was to assess the long-term effects of sucrose access on an object-in-place recognition task. This task consists in exposing rats to objects that occupy particular locations and then assessing their memory for this configuration by changing the locations occupied by the objects. Object-in-place recognition memory has been shown to be disrupted in patients with Alzheimer's disease (Fowler et al. 2002) and schizophrenia (Wood et al. 2002; Burglen et al. 2004). Specific lesions of the hippocampus and interconnected cortical regions, such as the PFC and perirhinal cortex have been shown to impair object-in-place memory (Barker and Warburton 2008, 2013).

The final aim was to assess the effect of sucrose consumption during adolescence on the density of parvalbumin-containing $\gamma$-aminobutyric acid (GABA)-ergic interneurons in the prefrontal cortex and hippocampus. Expression of parvalbumin-expressing GABAergic interneurons are integral to higher-order forms of behavioral control and have been implicated in attentional setshifting (Bissonette et al. 2014) and working memory (Timofeeva and Levin 2011). These cognitive functions are mediated by the dorsolateral PFC, and deficits in these various high-order cognitive processes are also core features of neuropsychiatric diseases including schizophrenia (Curley and Lewis 2012; Lewis et al. 2012). Therefore, we sought to determine whether intermittent access to sucrose during adolescence leads to long-lasting changes in their level of expression.

\section{Results}

\section{Sucrose consumption}

Rats provided with daily access to sucrose consumed significantly greater amounts over time $\left(F_{(3,9)}=49.9, P<0.001\right)$. The amount consumed (grams) per kilogram body weight differed across weeks $\left(F_{(3,12)}=4.672, P<0.05\right)$ and was significantly greater in week 3
A

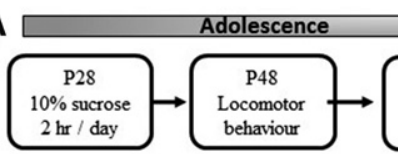

B

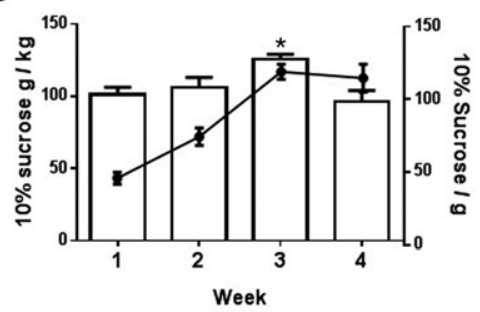

D

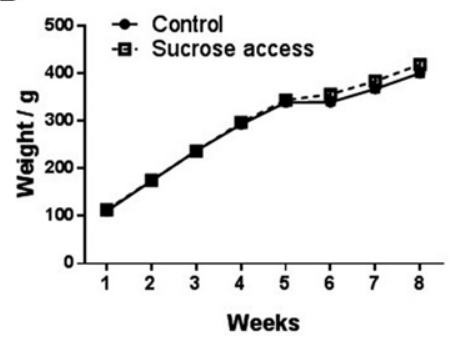

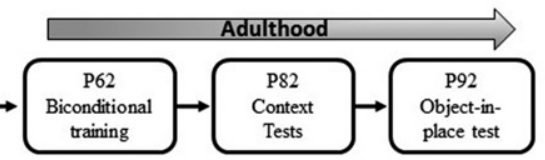

C

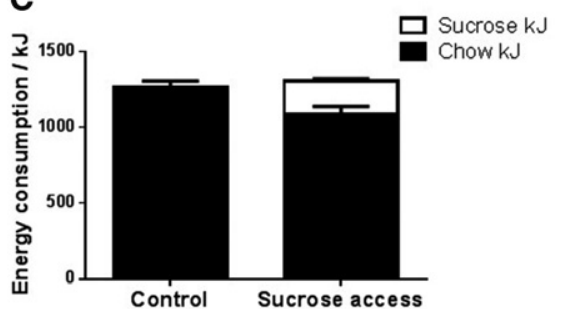

E

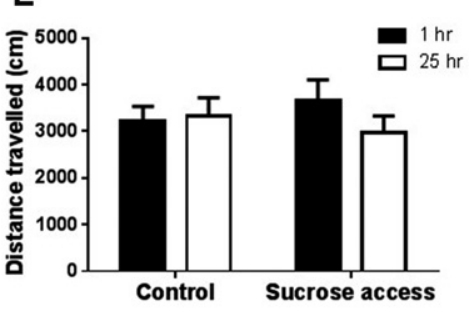

Figure 1. (A) Time line of experimental manipulations. (B) Line graph illustrates sucrose intake per cage of four rats each week (gram solution/cage/day) while the histogram reports gram sucrose consumed per kilogram body weight. (C) Mean 24-h energy consumption (kilojoules) per cage of four rats in week 4 (chow in solid and sucrose in open bar). (D) Mean weights of rats in control and sucrose access groups. (E) Mean distance traveled during 20-min locomotor testing. Error bars represent SEM, Control $N=16$, Sucrose $=16 .\left({ }^{*}\right) P<0.05$. 
of access $(t=3.573, P<0.01)$ as shown in Figure $1 \mathrm{~B}$. Sucrose consumed per body weight (grams/kilograms) was calculated by dividing the mean weekly sucrose consumption (grams per cage) by the total weight of the rats in the cage (kilograms). Total energy intake between sucrose and control rats did not differ $(t<1)$ as shown in Figure 1C. All rats gained weight over time $\left(F_{(7,259)}=\right.$ 2089.0, $P<0.001$ ), and the sucrose exposed rats were significantly heavier as shown in Figure 1D (main effect group: $F_{(1,37)}=5.2, P<$ 0.05 , group $\times$ time interaction $\left.F_{(7,259)}=9.3, P<0.001\right)$. Sucrose exposed rats were significantly heavier from week 6 onward ( $F^{\prime}$ s > 8.0). Furthermore, postmortem dissection of retroperitoneal and gonadal fat pads showed that sucrose fed rats had significantly greater white adipose tissue deposits than control rats (means [SEM] Control: $7.8 \mathrm{~g}$ [0.47], Sucrose: $10.1 \mathrm{~g}$ [0.46] $P=$ 0.001).

\section{Locomotor activity}

Analysis of the distance traveled (centimeters) shown in Figure $1 \mathrm{E}$ revealed that locomotor activity did not differ between rats with or without a history of sucrose or between rats provided with access 25 or $1 \mathrm{~h}$ before testing ( $F^{\prime} \mathrm{s}<1$ ), and there was no significant interaction between groups and time between sucrose exposure and test $\left(F_{(1,28)}=1.074, P=0.31\right)$.

\section{Acquisition of conditional discriminations}

Three rats in the sucrose group and four in the control group failed to acquire the visual discrimination (no consistent preference for the correct lever across all eight blocks of training, and during the final block of training prior to test the rats pressed the incorrect lever more than the correct lever) and were excluded from all behavioral analyses.

The remaining rats in each group acquired the visual and auditory conditional discrimination tasks, as shown by their preference for the correct lever $\left(F_{(1,23)}=155.9, P<0.001\right)$ during the unreinforced CS1 period, and there were no significant between-group differences in acquisition or group $\times$ repeated measures interaction, $F^{\prime} \mathrm{s}<1$ ). There was an overall increase in responding across the blocks of training $\left(F_{(7,161)}=\right.$ 8.4, $P<0.001)$, indicating progressively greater preference for the correct lever (lever $\times$ session interaction, $F_{(7,161)}=$ $19.8, P<0.001)$.

\section{Extinction test performance}

Rats received test sessions in which compounds of the training stimuli were presented under extinction. These compounds combined stimulus elements that dictated either the same ("congruent") or different ("incongruent") instrumental responses during initial training. The mean response rates (correct vs. incorrect) for each of the three trial types (single element, congruent, and incongruent) were analyzed.

\section{Congruent performance}

As shown in Figure 2A, rats made significantly more responses to the correct than incorrect lever during the congruent compound presentations $\left(F_{(1,23)}=\right.$ $66.4, P<0.001)$. There were no signifi- cant differences between the groups, $(F<1)$, or a significant group $\times$ lever interaction $\left(F_{(1,23)}=2.55, P=0.124\right)$.

\section{Incongruent performance}

Consistent with previous reports (Haddon and Killcross 2006; Reichelt et al. 2013a; Nelson et al. 2014), control rats used contextual cues to disambiguate the conflicting response information, responding according to the stimulus element that had previously been trained in that same test context (Fig. 2B). However, rats with a history of sucrose exposure did not make more responses to the correct than incorrect lever during the incongruent compound. Analysis revealed no significant effects of group $(F<1)$ or lever $\left(F_{(1,23)}=1.262, P=0.273\right)$ but, importantly, the interaction between group and lever was significant $\left(F_{(1,23)}=4.909, P<0.05\right)$. Inspection of the simple effects of the group $\times$ lever interaction revealed a significant effect of lever in control rats $\left(F_{(1,23)}=\right.$ $6.332, P<0.05)$; however, there was no significant effect of lever in rats with a history of sucrose exposure $(F<1)$.

\section{Single stimulus elements}

Both groups showed accurate conditional discrimination performance when single training stimulus elements were presented (Fig. 2C), i.e., when tested on the stimulus elements acquired during training and when there was no conflict. Consequently, both groups produced more correct responses $\left(F_{(1,23)}=43.7, P<0.001\right)$ with no effect of group or interaction (both $F<1$ ).

\section{Magazine activity}

No significant differences were observed in overall magazine responding $(F<1)$ (mean entries per min [ \pm SEM] Control: 8.1 [2.5]; Sucrose access: 8.9 [2.1]). When responding to stimuli was examined separately (mean entries per min $[ \pm$ SEM] Control: Congruent 6.3 [2.0], Incongruent 8.3 [2.4], Single element 8.5 [2.6]; Sucrose access: Congruent 7.6 [2.0], Incongruent 7.8 [1.8], Single element 9.6 [2.4]) a significant difference between stimulus
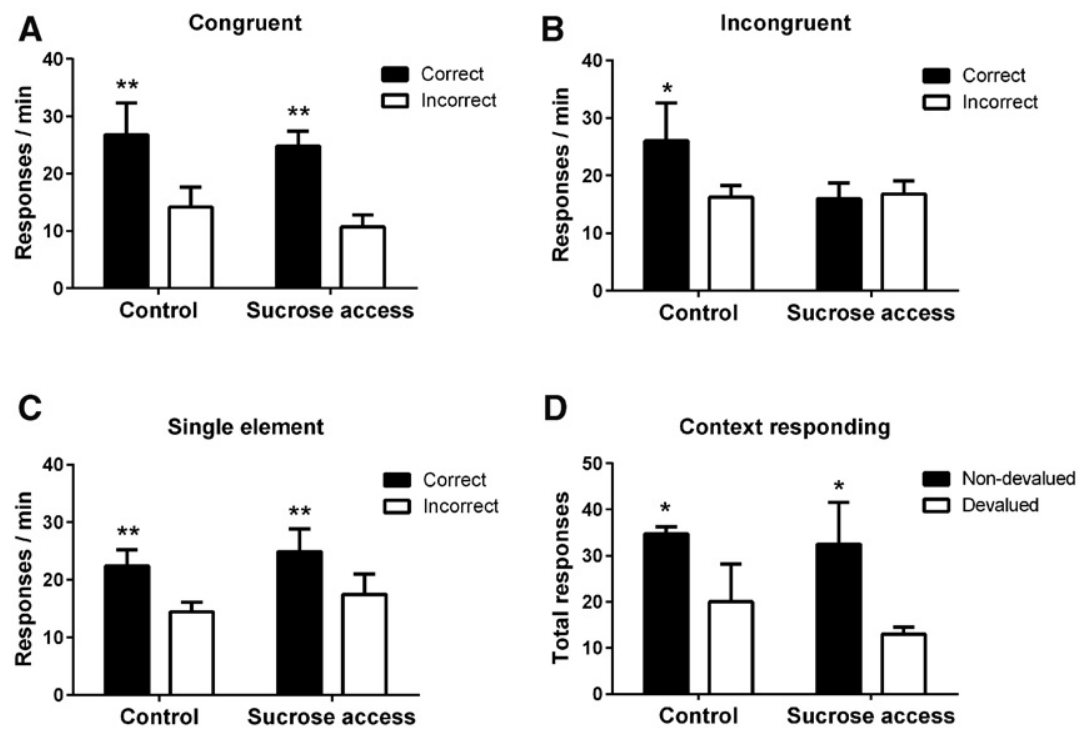

Figure 2. Test levels of lever pressing during $(A)$ congruent, $(B)$ incongruent compound, and $(C)$ single-element presentations in control and sucrose access rats. Data presented as $\log -1(x-1)$ of mean transformed data. $(D)$ Instrumental responding in the contexts associated with the nondevalued (black bars) and devalued (open bars) food rewards. Error bars represent SEM, Control $N=12$, Sucrose $N=13 .\left({ }^{*}\right) P<0.05,(* *) P<0.01$. 
types was observed $\left(F_{(2,46)}=3.4, P<0.05\right)$, indicating that the single elements provoked more magazine entries than the congruent and incongruent compounds, but there was with no effect of group or interaction (both $F<1$ ).

\section{Context devaluation}

To determine whether there were any differences between the groups in their ability to discriminate the two test contexts, we assessed the effect of outcome prefeeding on instrumental responding in the contexts. Rats from each group consumed comparable amounts of the reward pellet outcomes (mean [ \pm SEM] Control: $10.7 \mathrm{~g}$ [0.7]; sucrose access $11.4 \mathrm{~g}$ [0.5]). Both groups (Fig. 2D) displayed intact context-outcome associations as they made fewer lever-press responses during extinction when prefed the outcome that had previously been earned in that test context compared with when prefed the outcome associated with the other context $\left(F_{(1,23)}=7.77, P=0.01\right)$. There was no effect of group or any interaction with group (both $F<1$ ). Magazine entries did not significantly differ $(F<1)$ between groups (mean [ \pm SEM] Control: devalued 11.3 [2.4]; nondevalued 9.4 [1.4]; Sucrose access: devalued 11.5 [3.0]; nondevalued 9.5 [2.1]) and there was no overall effect of devaluation $\left(F_{(1,23)}=1.4, P=0.251\right)$

\section{Object-in-place memory}

Exploration times during the familiarization phase did not differ between the control and sucrose access groups $(F<1)$ (mean $[ \pm$ SEM]: Control $=50.9 \mathrm{sec}[3.1]$; Sucrose $=52.4 \mathrm{sec}[4.8])$. At test, the discrimination ratios significantly differed between control and sucrose groups $(t=9.33, \mathrm{df}=30, P<0.001)$, such that control rats spent more time exploring the moved objects (B and $\mathrm{C}$ ) than the ones that remained in the same location (A and D), whereas sucrose access rats spent just as much time exploring $\mathrm{B}$ and $\mathrm{C}$ as they did A and D (Fig. 3B). Furthermore, the total amount of time spent exploring the objects during the test phase (mean [ \pm SEM] Control: $41.6 \mathrm{sec}$ [3.3], Sucrose access: $32.5 \mathrm{sec}$ [3.4]) did not significantly differ between groups $(t=1.93, \mathrm{df}=$ $30, P=0.064$ ). These results show that rats in both groups spent similar amounts of time exploring but differed in how this time was distributed across the objects, indicating that the impairment among the sucrose exposed rats was not due to a change in the motivation to explore.

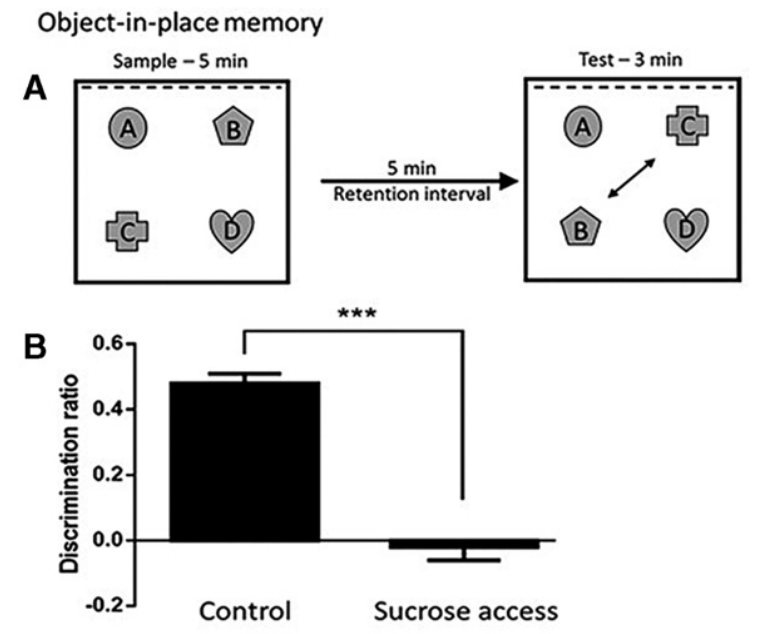

Figure 3. (A) Schematic of the object-in-place memory task. (B) Mean discrimination ratio $(d 2)$ at test in control and sucrose access rats. Error bars represent SEM, Control $N=16$, Sucrose $N=16$. $\left(^{* * *}\right) P<0.001$.

\section{Parvalbumin immunohistochemistry}

Rats exposed to intermittent sucrose throughout adolescence had significantly fewer parvalbumin immunopositive cells in the hippocampus, both overall $\left(F_{(1,29)}=27.6, P<0.001\right)$ and in its subregions (CA1: $F_{(1,29)}=21.3, P<0.001$; CA3: $F_{(1,29)}=12.3$, $P=0.001$; dentate gyrus: $\left.F_{(1,29)}=13.1, P=0.001\right)$ as shown in Figure 4A,B. Rats exposed to intermittent sucrose throughout adolescence also had significantly fewer parvalbumin immunopositive cells in the mPFC overall $\left(F_{(1,29)}=10.4, P=0.003\right)$ and in the prelimbic $(\operatorname{PrL}) \operatorname{PFC}\left(F_{(1,29)}=8.359, P<0.01\right)$, but not in the infralimbic (IL) PFC $\left(F_{(1,29)}=3.97, P=0.056\right)$; as shown in Figure $5 \mathrm{~A}, \mathrm{~B}$.

\section{Discussion}

This experiment exposed young rats to a 10\% sucrose solution for a limited period of time each day across their adolescence and examined their subsequent performance on tasks that required the use of (1) contextual information to disambiguate response conflict and (2) of spatial relations between objects to identify what has changed in the environment. Conflict control was examined by simultaneously presenting pairs of stimuli that had each controlled opposing ("incongruent") responses due to their initial conditional discrimination training in different contexts. Consistent with previous reports (Haddon and Killcross 2006; Marquis et al. 2007; Reichelt et al. 2013a), control rats were able to use contextual information to resolve this conflict task. Rats that had been exposed to intermittent sucrose across adolescence learned the biconditional discriminations just as well as control rats, but, in contrast to the control rats, failed to use the contextual information to discriminate between the context-appropriate and context-inappropriate levers during incongruent trials. No differences were observed in latency to make responses during the discriminations, suggesting that the performance deficit to incongruent compounds in the sucrose exposed rats was not due to increased impulsivity. Furthermore, the observation that daily limited exposure to sucrose across adolescence exerts longterm effects upon PFC function in the form of impairments in response-conflict resolution further extends the known effects of high sucrose diet consumption beyond that of the hippocampus. This conclusion derives from the finding that rat hippocampal lesions can enhance, rather than disrupt, incongruent-trial performance on the present Stroop-based task (Haddon and Killcross 2007) potentially due to the hippocampus not being required to utilize contextual cues as a task-setting cue to guide responding. However, PFC-dependent control appears to be best characterized as an explicit goal-directed process in which contextual or task-setting cues are used as the basis of higher-order rules to enable the appropriate control of choice responses (Haddon and Killcross 2007). Thus, it is possible that high sucrose diet in adolescence may exert long-term adverse effects on a variety of neural structures involved in high-order cognitive control of behavior, including the PFC.

We used devaluation of one outcome to assess whether the sugar intake across adolescence had impaired subsequent ability to discriminate between contexts, thereby impairing the use of contextual information to solve response conflict in the Strooplike task (Bar and Aminoff 2003). However, we found that rats that had received sucrose across their adolescence showed greater instrumental responses in the context associated with the nondevalued outcome than in the context associated with the now devalued outcome. This indicates that the history of sucrose had not impaired the subsequent ability to use contextual information to guide responding in a goal-directed manner. This is a novel finding, as previous studies have provided evidence that goal-directed instrumental actions are disrupted in rats provided with $2 \mathrm{~h}$ daily 
A

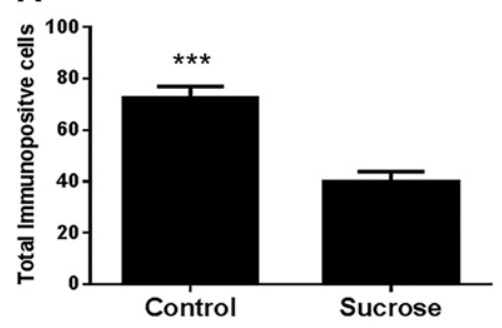

C
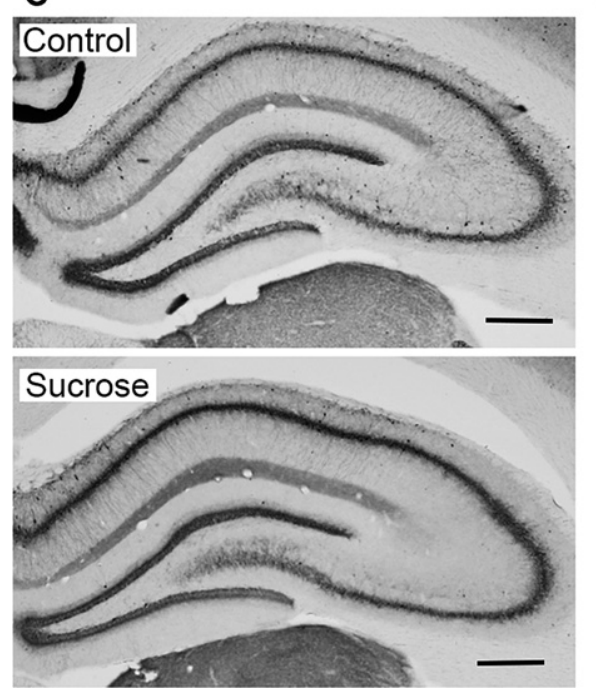

B

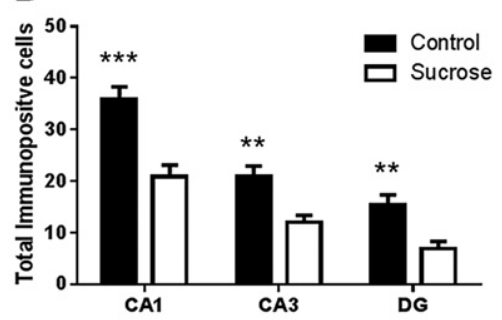

D
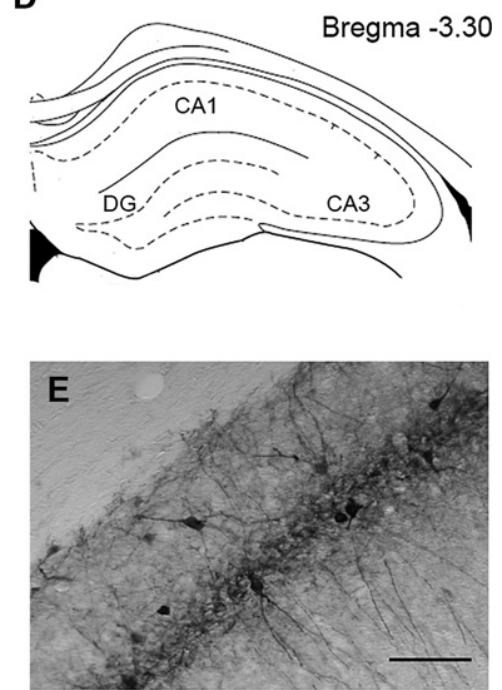

Figure 4. Results of parvalbumin immunohistochemistry in dorsal hippocampus. $(A)$ Mean number of parvalbumin immunopositive cells in control and sucrose exposed rats in the hippocampus overall (A) and in the hippocampal subfields CA1, CA3, and dentate gyrus (B). (C) Photomicrographs show representative hippocampal staining of control and sucrose exposed rats, $4 \times$ magnification, marker represents $250 \mu \mathrm{m}$. (D) Regions of the hippocampus studied. (E) Photomicrograph showing parvalbumin stained neurons in the CA1 of a control rat at $20 \times$ magnification, marker represents $25 \mu \mathrm{m}$.

ies indicating goal-directed instrumental behavioral deficits following sucrose consumption, it is possible that the expression of frontostriatal D2 receptors are chronically down-regulated by daily restricted access to sucrose consumption. This suggestion warrants further exploration by analysis of dopamine receptor mRNA and potentially through administration of D2 receptor agonists to ameliorate cognitive deficits.

Rats exposed to sucrose for $2 \mathrm{~h}$ a day during adolescence also exhibited a subsequent deficit in an object-in place memory task. In this task, rats are first exposed to four objects, A, B, C, and D occupying distinct locations in a familiar environment. After a brief retention interval, they are reexposed to the objects, two of which, B and C, now occupy each other's previous locations, while the other two, A and D, continue to occupy their original locations. Control rats spent more time exploring the objects whose locations were changed, B and C, than the objects that remained in the same locations. In contrast, rats exposed to sucrose across adolescence spent just as much time exploring both pairs of objects. Previous studies have provided evidence that this object-in-place recognition memory involves several brain regions. These include the hippocampus, which codes spatial relations between the objects (Barker and Warburton 2011), the perirhinal cortex, which constructs a representation of the identities access to a high fat/high sugar condensed milk solution (Furlong et al. 2014 ) or to a $10 \%$ sucrose solution (Kendig et al. 2013), suggesting that $2 \mathrm{~h}$ daily access to these beverages accelerates the development of habitual instrumental behaviors compared with rats with constant sucrose access. Acceleration of habitual responses has also been observed following sensitization of the dopaminergic system by d-amphetamine (Nelson and Killcross 2006). Previous studies have indicated that frequent intake of high-fat and high-sugar foods is associated with decreased dopamine D2-receptor sensitivity and expression in the striatum of rodents and obese humans, paralleling the effects seen after chronic exposure to psychostimulants (Bello et al. 2002, 2003). D2 receptors are implicated in controlling behavioral flexibility (Yawata et al. 2012) and their down-regulation therefore could contribute to loss of goal-directed control.

Thus, in light of our observations that sucrose consumption impairs behavioral flexibility and previous stud-
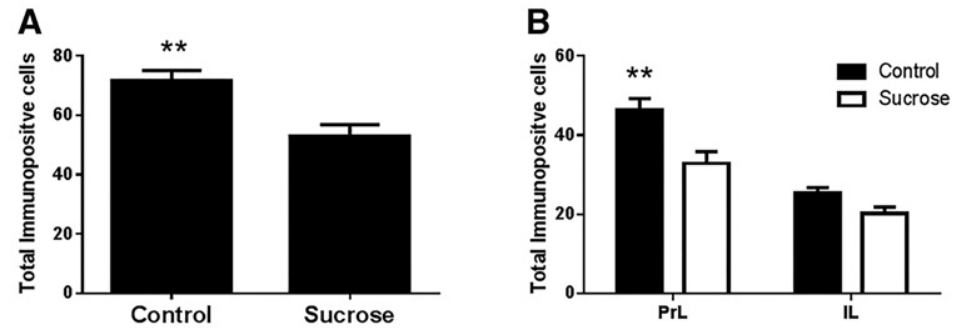

C

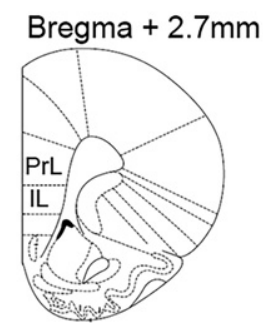

D
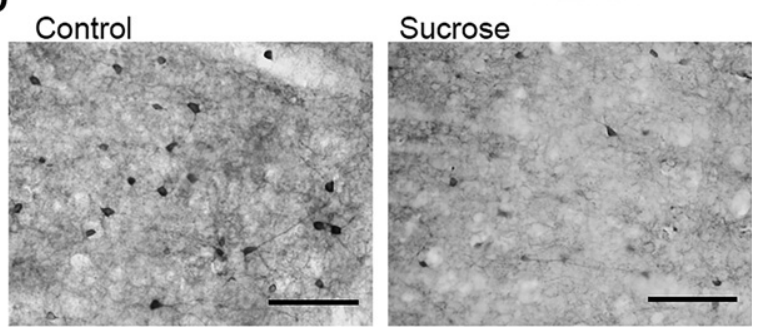

Figure 5. Results of parvalbumin immunohistochemistry in PFC. (A) Mean number of parvalbumin immunopositive cells in control and sucrose exposed rats in the PFC overall (A) and in the PFC subfields prelimbic PFC (PrL) and infralimbic PFC (IL) (B) Photomicrographs show representative PFC staining of control and sucrose exposed rats. (C) Regions of the mPFC studied. (D) Photomicrographs show representative parvalbumin stained neurons in the PrL PFC of a control rat and sucrose exposed rat $20 \times$ magnification, marker represents $25 \mu \mathrm{m}$. 
of the objects (Barker et al. 2007), the mPFC, which may integrate the spatial and object information (Kesner and Ragozzino 2003; Barker et al. 2007), as well as interactions between these regions, especially, those between hippocampus and the mPFC (Barker and Warburton 2011). The object-in-place memory deficit observed in sucrose exposed rats could not be attributed to hyperactivity, as the sucrose fed rats did not differ in their levels of locomotor behavior from control rats, nor to a lack of motivation to explore as the sucrose fed rats did not differ in the total time spent exploring from control rats.

Immunohistochemical analysis performed $8 \mathrm{wk}$ after termination of the intermittent sucrose access revealed a significant deficit in parvalbumin-immunoreactive cell density in the hippocampus and PFC. Consistent with these findings, other studies have reported correlations between reductions in parvalbumin expression and impairments in fear conditioning (Courtin et al. 2014) and attentional set formation (Bissonette et al. 2014). Studies in postmortem schizophrenia patients and rodent models have also demonstrated a reduction in parvalbumin expression in the mPFC (Lewis et al. 2005) and the hippocampus (Lodge et al. 2009; Konradi et al. 2011). A recent study by Boley et al (2014) indicated that a lentiviral delivered short hairpin RNA-induced knockdown of parvalbumin expression in the ventral hippocampus in rats led to downstream increases in spontaneous dopamine neuron population activity in the VTA and an enhanced locomotor response to amphetamine administration. Sugar-induced alteration of the dopamine system has been previously reported in rats maintained on a daily 20-min sugar-feeding schedule, this alteration included increased dopamine turnover in the nucleus accumbens (Hajnal and Norgren 2002) and up-regulation of DA transporter mRNA in the ventral tegmental area and nucleus accumbens (Bello et al. 2002, 2003). Dopamine is critical for modulating cortical GABA mediated inhibition and glutamate mediated excitation in the PFC and other cortical regions ( $\mathrm{O}^{\prime} \mathrm{Don}$ nell 2010). This dopamine directed control of the excitationinhibition balance within the cortex and hippocampus is refined during adolescence (Tseng and O'Donnell 2007; O'Donnell 2010). Thus, alterations in dopamine turnover and activity may contribute to the observed loss of parvalbumin-expressing GABA-interneurons, leading to alterations in the maturation of circuits during adolescence that are responsible for excitation-inhibition balance in the PFC, leading to cortical disinhibition and behavioral impairments (O'Donnell 2011).

Taken together, these findings indicate that perturbations in parvalbumin expression are associated with cognitive deficits and alterations in dopamine signaling. These findings also provide additional support for the suggestion that sucrose access during adolescence can produce enduring neuropathological changes in the rat and that these changes underpin our observed deficits in response conflict resolution and object-in-place recognition memory. However, further studies are needed to establish the extent of changes, whether these extend to other regions involved in reward learning and behavioral flexibility, and, indeed, whether such changes are causally related to the cognitive deficits observed here.

High-sugar diets have been implicated in weight gain and obesity, by increasing energy consumption to an extent that exceeds energy output, thereby distorting mechanisms controlling energy balance (DiMeglio and Mattes 2000). Behavior was tested 6 wk after sucrose exposure ceased, thus it is likely that any effects on blood sugar had dissipated at this time. Blood glucose levels were not measured in this study, however it has recently been shown that rats exposed to $10 \%$ sucrose continuously for $30 \mathrm{~d}$ had a decreased ability to tolerate an oral glucose load (Sheludiakova et al. 2012). Furthermore, a recent study indicated that plasma insulin levels remained elevated after sucrose access ceased in adolescent, but not adult sucrose exposed rats (Hsu et al. 2015).

Importantly, the impairments in cognitive control observed in our current study indicate that sucrose access for a limited period each day can induce subsequent deficits in decision-making and behavioral flexibility. The present results may have implications for mental health and neurodevelopmental disorders although it must be acknowledged that a range of variables contribute to mental health in people and that disorders of mental health have a complex atiology. Nevertheless, the finding that daily consumption of sucrose in rats led to various cognitive deficits provides support for the proposal that sugar consumption in children and adolescents may likewise have adverse effects on cognitive development. The World Health Organisation's guideline for sugar intake is $\sim 25 \mathrm{~g}$ per day ( $10 \%$ of total energy intake), but a single can of standard soft drinks may contain as much as $40-50 \mathrm{~g}$ of sugar. There is growing evidence that the pathophysiology of many mental disorders might emerge from maturational aberrations of normal neurodevelopmental changes in the adolescent brain, particularly in the PFC (Paus et al. 2008). Thus, high levels of sugar-sweetened beverages consumed during adolescence may pose a risk factor for the development of mental health disorders, perhaps by impairing neurocognitive functions affecting decision-making and memory.

\section{Materials and Methods}

\section{Subjects}

Male albino Sprague Dawley rats (Animal Resources Centre, Western Australia) were used $(n=32)$. Rats arrived in the laboratory at $3 \mathrm{wk}$ of age (mean weight $80 \mathrm{~g}$ ). They were housed in plastic cages $26 \times 40 \times 60 \mathrm{~cm}$, with four rats per cage in a colony room that was on a 12-h light-dark cycle (lights on at 07:00), maintained at a temperature of $21 \pm 2^{\circ} \mathrm{C}$ and a humidity of $55 \pm 5 \%$. Rats were acclimated to the laboratory for $7 \mathrm{~d}$ during which they were handled each day. Training and testing occurred in the light phase between 09:00 and 17:00. The experimental procedures were approved by the University of New South Wales Animal Ethics Committee in accordance with the Animal Code of Practice for the Care and Use of Animals for Scientific Purposes.

\section{Sucrose access}

Figure $1 \mathrm{~A}$ illustrates the experimental time line. Rats were acclimated to their housing conditions for $7 \mathrm{~d}$ (P21-P27) prior to access to a $10 \%$ sucrose solution. From P28 rats in the sucrose access condition $(N=16)$ were permitted $2 \mathrm{~h}$ access in their home cage to $200 \mathrm{~mL}$ of $10 \%$ sucrose (w/vol; CSR white sugar). Fresh sucrose was prepared daily and was available between 8.00 a.m. and 10.00 a.m. for 28 d until P56. The caloric density of the sucrose $(1.7 \mathrm{~kJ} / \mathrm{mL})$ was similar to that of commonly available sugar-sweetened beverages. Consumption was recorded by weighing the bottles before and after the $2 \mathrm{~h}$ sucrose access period. All rats had ad lib access to chow and water at all times. At P58 rats were food restricted. This consisted in providing each home cage with a total of $60 \mathrm{~g}$ ( $\sim 80 \%$ of regular intake) of chow. Water continued to be available ad libitum.

\section{Locomotor behavior}

Locomotor behavior was assessed on P48, following $20 \mathrm{~d}$ of exposure to sucrose. We assessed whether access to sucrose affected locomotor activity; we also tested whether any such effect was influenced by the time between the last sucrose access and test by examining rats at 1 and $25 \mathrm{~h}$ post sucrose. This was undertaken in both control rats and those in the sucrose access group. Specifically, half of the rats in each group (control $[N=8]$ and sucrose $[N=8]$ ) were provided with $2 \mathrm{~h}$ of sucrose access in their home cages $25 \mathrm{~h}$ prior to testing; the other half of the rats in each group (control $[N=8]$ and sucrose $[N=8]$ ) received $2 \mathrm{~h}$ 
access to sucrose $1 \mathrm{~h}$ prior to testing. Locomotor activity was assessed in four identical square arenas $(43.2 \mathrm{~cm}$ height $\times 43.2$ $\mathrm{cm}$, Med Associates) located in sound-attenuating chambers (Med Associates). Each arena was equipped with three 16 beam infrared arrays located on both the $X$ and $Y$ axes for positional tracking and $Z$ axis for rearing detection. The duration of the test was 20 min.

\section{Biconditional discrimination}

\section{Stimuli and apparatus}

Biconditional instrumental training took place from P62 in eight chambers (Med Associates) each measuring $30 \mathrm{~cm}$ wide, $21 \mathrm{~cm}$ high and $24 \mathrm{~cm}$ deep, located in sound-attenuating boxes (Med Associates) that were arranged in a two-by-four array in a room which remained dark throughout the experiment. The ceiling, door, and back wall of each chamber were clear Perspex and the left and right walls were stainless steel. The floor of each chamber was constructed of 19 stainless steel rods $(4.8 \mathrm{~mm}$ in diameter, spaced $16 \mathrm{~mm}$ apart). In four of the chambers, the floor remained as stainless steel rods (Context 1 ), in the other four a stainless steel mesh was located on the stainless steel rods (Context 2). The right hand side wall of the chambers contained a recessed magazine where grain or sucrose pellets ( $45 \mathrm{mg}$; P.J. Noyes) were delivered. Two retractable levers were located to the left and right of the magazine. Above each lever was a $2-\mathrm{cm}$ diameter panel light. Entries to the magazine were detected by an infrared sensor and the roof of the magazine contained a green LED light. A $3 \mathrm{~W}$ house light was located at the top center of the left wall. Auditory stimuli consisted of a $2-\mathrm{kHz}$ tone and $10-\mathrm{Hz}$ train of clicks, both delivered through speakers in the left wall at $75 \mathrm{db}$. Visual stimuli consisted of either illumination of the two panel lights plus illumination of the magazine light, or illumination of the house light. A computer equipped with MED-PC software (version IV; Med Associates Inc.) controlled the experimental events and recorded data.

\section{Behavioral procedure}

\section{Pretraining}

Rats received two 48-min sessions of magazine training. Food pellet rewards (grain or sucrose) were delivered on a random time 120 -sec schedule. Magazine training occurred in a morning session in one context (e.g., rod floors with grain pellets) and, $3 \mathrm{~h}$ later, an afternoon session in the alternative context (e.g., mesh floors and sucrose pellets). This allocation of contexts and their associated outcome remained consistent throughout training and was counterbalanced across animals (see below). After magazine training, the rats were trained to respond during alternating presentations of the left and right levers. Each session lasted 48 min and consisted of twenty-four $60 \mathrm{sec}$ trials (12 each of the left and right lever presentation). Rats received two training sessions per day, one in the morning and the other, $3 \mathrm{~h}$ later, in the afternoon. On the first day lever pressing was reinforced on a continuous schedule (CRF) and on the second day was reinforced on a random interval 15-sec schedule (RI15). Rats then progressed to biconditional discrimination training.

\section{Biconditional discrimination training}

Rats were trained on two simultaneous biconditional discriminations (see Table 1). All elements were counterbalanced across rats. For half the rats, the floor of context 1 (C1) consisted of stainless steel rods, while the floor of context 2 (C2) consisted of stainless steel mesh; for the other half of the rats, the mesh floor was C1 and the floor with rods was C2. Previous studies (Reichelt et al. 2013b) have shown that these contexts with their distinct floors are highly discriminable for rodents. Half of the rats in each of these allocations received the auditory discrimination in $\mathrm{C} 1$ and the visual discrimination in $\mathrm{C} 2$, and this allocation was reversed for the other half of each subset of rats. During the auditory discrimination rats received presentations of auditory (A) cues
Table 1. Experimental design indicating biconditional training and test stimuli

\begin{tabular}{llccc}
\hline & & \multicolumn{3}{c}{ Test sessions } \\
\cline { 3 - 5 } Context & $\begin{array}{l}\text { Biconditional } \\
\text { training }\end{array}$ & $\begin{array}{c}\text { Congruent } \\
\text { probe }\end{array}$ & $\begin{array}{c}\text { Incongruent } \\
\text { probe }\end{array}$ & $\begin{array}{c}\text { Single } \\
\text { element } \\
\text { reinforced }\end{array}$ \\
\hline C1 & A1: LP1 $\rightarrow$ O1 & A1V1 & A1V2 & A1, A2 \\
C2 & A2: LP2 $\rightarrow$ O2 & A2V2 & A2V1 & V1, V2 \\
& V1: LP1 $\rightarrow$ O1 & A1V1 & A1V2 & \\
\hline & V2: LP2 $\rightarrow$ O2 & A2V2 & A2V1 & \\
\hline
\end{tabular}

$\mathrm{C} 1 / \mathrm{C} 2, \mathrm{O} 1 / \mathrm{O} 2, \mathrm{LP} 1 / \mathrm{LP} 2, \mathrm{~A} 1 / \mathrm{A} 2$, and V1/V2 refer to different experimental chambers (contexts), outcomes, levers, and auditory and visual stimuli respectively. The specific schedule of events was counterbalanced across animals.

(tone or clicker [A1 or A2]) during which the correct lever-press responding (left or right [LP1 or LP2], counterbalanced) led to an outcome (O1; food pellets). During the visual discrimination lever-press responding (LP1 and LP2, counterbalanced) led to reward (O2; sucrose pellets) in the presence of visual cues (V) (illumination of the house light, or two stimulus lights plus the magazine light [V1 and V2]). Rats received one morning session in $\mathrm{C} 1$ and one afternoon session in $\mathrm{C} 2$.

Each session lasted $48 \mathrm{~min}$ in total and consisted of 24 trials (six of each trial type; A1 and A2 or V1 and V2) with a variable intertrial interval (range $40-60 \mathrm{sec}$; mean, $50 \mathrm{sec}$ ). Stimuli were presented for $60 \mathrm{sec}$, during which both levers were present. Reinforcement was unavailable for the first $10 \mathrm{sec}$ (CS1) and was then available during the final 50 -sec period (CS2) on the RI15 schedule. The levers were retracted at the end of the $60 \mathrm{sec}$ stimulus presentation and remained retracted during the intertrial interval. Rats received 16 sessions of each stimulus condition, two sessions per day, for $16 \mathrm{~d}$.

\section{Test sessions and probe stimuli}

Test stimuli were congruent and incongruent stimulus compounds or single training stimulus elements previously associated with each training context as described previously (Reichelt et al. 2013a). For example, if C1 was previously associated with auditory stimuli A1 and A2, these single stimulus elements were presented during the test; in the alternative context (C2) visual stimuli V1 and V2 were presented as single elements. The compound test stimuli were categorized as either congruent or incongruent depending on the responses previously required during training. Congruent stimulus compounds were composed of stimulus elements which had each signaled the same lever-press responseoutcome relation during training on the biconditional discrimination task. Thus, the elements previously presented in A1V1 and A2V2 compounds had each signaled the same lever-press responses in training (LP1 and LP2, respectively). Incongruent stimulus compounds, A1V2 and A2V1 were composed of stimulus elements which had signaled different, and thus conflicting, lever-press responses during biconditional discrimination training. "Correct" responding to incongruent compounds is defined by responding according to the stimulus element previously trained in the appropriate context. Thus, if during the test session the context had been previously associated with the visual discrimination, the visual stimulus element (V1 or V2) of the incongruent compound (A1V2 or A2V1) was the correct cue. For example, if presentations of A1V2 occurred in the context where A1 had been presented during the biconditional training, selection of the lever appropriate to A1, rather than $\mathrm{V} 2$, was deemed to be correct. Therefore, for the rats to respond correctly to the incongruent stimulus element compounds they must utilize contextual information provided by the test context to disambiguate responding.

Following acquisition of the biconditional discrimination, rats received probe test trials within training sessions. Rats 
received two test sessions per day, one in each context. Test sessions consisted of four extinction probe trials consisting of presentations of congruent and incongruent audio-visual compounds of training stimuli (A1V1, A2V2, A1V2, A2V1) and eight reinforced training trials of single training elements associated with the context (A1, A2 or V1, V2), presented in a manner identical to training trials. Test session duration was $24 \mathrm{~min}$ and trial order was randomized; each extinction probe trial was presented once during the test session. Testing took place over $4 \mathrm{~d}$.

\section{Analysis}

To assess whether the history of sucrose access selectively disrupted incongruent, but not congruent compound performance, within-subjects ANOVAs were performed on congruent and incongruent compound responses separately. As the variance of test data was found to increase with the mean, data were subject to a logarithmic transformation $\left(\log _{10}(x+1)\right)$ to correct for the heterogeneity of variance before performing any further analyses (Howell 2013).

\section{Selective reward devaluation on contextual conditioning}

Rats prefed one food reward (i.e., grain or sucrose pellets) to satiety show a selective reduction in instrumental responding for the sated reward associated with that particular context (Haddon and Killcross 2006; Reichelt et al. 2013b). To assess whether the sucrose history influenced this contextually specific devaluation effect, we subjected rats to two 10-min extinction sessions during which both levers were available. Prior to each extinction session, the rats received 40-min free access to one of the two rewards (20 g of sucrose or grain pellets) earned during training. This access occurred in individual cages located in a separate room. Each rat was tested in both contexts (Context 1 and Context 2), with testing order (devalued versus nondevalued) counterbalanced. We expected that control rats would produce more leverpress responses in the context associated with the nondevalued outcome. The question of interest was whether those with a history of sucrose access would also behave in this outcome valuespecific manner.

\section{Object-in-place memory}

The object-in-place task is a procedure developed to test rodent memory that combines the elements of traditional measures of object recognition and object placement by interrogating memory for the recognition of objects together with the spatial context in which they occur (Barker and Warburton 2013). The apparatus consisted of an open-field arena $(60 \times 60 \times 60 \mathrm{~cm})$ constructed from black PVC plastic. One wall was covered in white opaque Perspex and served as a spatial cue (Good and Hale 2007). A video camera positioned directly above the arena recorded the behavior of each rat to a DVD. The room was dimly lit (25 Lux) by an overhead light. The objects used were commercial products (e.g., beer bottles, coffee mugs, water bottles, cans), made of a variety of materials (aluminium, glass, plastic, and ceramic) and varying in both height $(7.5-15 \mathrm{~cm})$ and width $(4.5-8 \mathrm{~cm})$. Rats were acclimatized to the empty arena for $10 \mathrm{~min}$ per day on two consecutive days, and testing commenced the next day.

Testing consisted in a familiarization phase followed by test. During the familiarization phase, each rat was placed into the middle of the arena with four different objects (A, B, C, D) located equidistantly from the center and allowed to explore for $5 \mathrm{~min}$. The rat was then removed for $5 \mathrm{~min}$ (retention interval) and the arena and objects were cleaned with $80 \%$ ethanol to remove odors. For the test phase, an identical set of four objects were placed in the arena, but the locations of $B$ and $C$ were switched (see Fig. 4A). The test phase was $3 \mathrm{~min}$ in duration. If rats remember the original configuration, they will spend more time exploring the two objects whose locations have been reversed (B and C) than the two objects that remain in the same locations (A and D).

\section{Analysis}

Exploration was defined as the rat's head within $2 \mathrm{~cm}$ of the object with the neck extended and vibrissae moving around the object. Other behaviors such as sitting on or resting against the object were not scored. Data were scored using Macropod ODlog software by the experimenter and a second observer naïve to group allocation. The correlation between the two observers was high $(r>$ 0.90). Data are reported as the Exploration Ratio, which was the difference between the time spent exploring the moved objects ( $\mathrm{B}$ and $\mathrm{C}$ ) - unmoved objects ( $\mathrm{A}$ and $\mathrm{D}$ ) divided by the time spent exploring all the objects (A, B, C, and D) $\left(t_{\text {moved }}-t_{\text {unmoved }}\right) /$ $\left(t_{\text {moved }}+t_{\text {unmoved }}\right)$. This discrimination ratio thus takes into account individual differences in the total amounts of time spent exploring (Ennaceur and Delacour 1988).

\section{Parvalbumin immunohistochemistry}

Immediately following the behavioral testing $(\sim 8 \mathrm{wk}$ after the termination of sucrose access), rats were deeply anesthetized with sodium pentobarbital $(100 \mathrm{mg} / \mathrm{kg}$, i.p.) and perfused transcardially with $150 \mathrm{~mL}$ of $0.9 \%$ saline, containing heparin (5000 IU/ $\mathrm{mL}$ ), followed by $400 \mathrm{~mL}$ of $4 \%$ paraformaldehyde in $0.1 \mathrm{M}$ phosphate buffer (PB), $\mathrm{pH}$ 7.4. Brains were post-fixed for $1 \mathrm{~h}$ in the same fixative and placed in $20 \%$ sucrose solution overnight. Brains were frozen and sliced to $40 \mu \mathrm{m}$ coronal sections. Sections $(40 \mu \mathrm{m})$ from the prefrontal cortex (bregma $+2.7 \mathrm{~mm}$ ) and dorsal hippocampus (bregma -3.5), determined using a rat brain atlas (Paxinos and Watson 2006) were stained for parvalbumin immunoreactivity. Sections were washed in 0.1M PBS and blocked with $5 \%$ normal horse serum diluted in $0.1 \mathrm{M}$ PBS containing $0.1 \%$ Triton X-100. After incubation at $4^{\circ} \mathrm{C}$ with a mouse monoclonal antibody against parvalbumin (Millipore) at a dilution of 1:10,000 in PBS for $48 \mathrm{~h}$, biotinylated secondary antibody (dilution 1:200) was added. Sections were processed by the Streptavidin-Horseradish peroxidase method (Vector Laboratories, UK) and peroxidase was visualized using 3', 3'-diaminobenzadine (DAB) intensified with nickel chloride. No immunoreactivity could be detected in control sections, in which the primary antibody was omitted from the staining protocol. Sections were mounted onto $4 \%$ gelatin-coated slides, dehydrated, cleared in histolene, and coverslipped with Entellan. All slides were coded and analyzed blind to treatment. Total PFC and hippocampal counts of parvalbumin-positive neurons were measured using ImageJ (v1.46; http://imagej.nih.gov/ij); within the PFC, the infralimbic (IL), and prelimbic (PrL) cortices were examined, and within the hippocampus, the CA1, CA3, and dentate gyrus (DG) were examined.

\section{Statistics}

Statistical comparisons were made using a repeated measures analysis of variance (ANOVA) or independent $t$-tests (two-tail), where appropriate (SPSS version 22, IBM). All statistical analyses used a significance level of 0.05 .

\section{Acknowledgments}

We thank Dr. Nicole Jones and Mr. Jonathan Teo for their assistance with immunohistochemistry. A.C.R. is the recipient of an Australian Research Council Discovery Early Career Research Award (DE140101071).

\section{References}

Ames SL, Kisbu-Sakarya Y, Reynolds KD, Boyle S, Cappelli C, Cox MG, Dust M, Grenard JL, Mackinnon DP, Stacy AW. 2014. Inhibitory control effects in adolescent binge eating and consumption of sugar-sweetened beverages and snacks. Appetite 81: 180-192.

Bar M, Aminoff E. 2003. Cortical analysis of visual context. Neuron 38: $347-358$.

Barker GR, Warburton EC. 2008. NMDA receptor plasticity in the perirhinal and prefrontal cortices is crucial for the acquisition of long-term object-in-place associative memory. J Neurosci 28: 2837-2844. 
Barker GR, Warburton EC. 2011. Evaluating the neural basis of temporal order memory for visual stimuli in the rat. Eur J Neurosci 33: 705-716.

Barker GR, Warburton EC. 2013. Object-in-place associative recognition memory depends on glutamate receptor neurotransmission within two defined hippocampal-cortical circuits: a critical role for AMPA and NMDA receptors in the hippocampus, perirhinal, and prefrontal cortices. Cereb Cortex 25: 472-481.

Barker GR, Bird F, Alexander V, Warburton EC. 2007. Recognition memory for objects, place, and temporal order: A disconnection analysis of the role of the medial prefrontal cortex and perirhinal cortex. J Neurosci 27: 2948-2957.

Basu S, Yoffe P, Hills N, Lustig RH. 2013. The relationship of sugar to population-level diabetes prevalence: an econometric analysis of repeated cross-sectional data. PLoS One 8: e57873.

Bello NT, Lucas LR, Hajnal A. 2002. Repeated sucrose access influences dopamine D2 receptor density in the striatum. Neuroreport 13: 1575-1578.

Bello NT, Sweigart KL, Lakoski JM, Norgren R, Hajnal A. 2003. Restricted feeding with scheduled sucrose access results in an upregulation of the rat dopamine transporter. Am J Physiol Regul Integr Comp Physiol 284: R1260-R1268.

Berkey CS, Rockett HR, Field AE, Gillman MW, Colditz GA. 2004. Sugar-added beverages and adolescent weight change. Obes Res 12: $778-788$.

Berridge KC. 2012. From prediction error to incentive salience: mesolimbic computation of reward motivation. Eur J Neurosci 35: 1124-1143.

Bissonette GB, Bae MH, Suresh T, Jaffe DE, Powell EM. 2014. Prefrontal cognitive deficits in mice with altered cerebral cortical GABAergic interneurons. Behav Brain Res 259: 143-151.

Boley AM, Perez SM, Lodge DJ. 2014. A fundamental role for hippocampal parvalbumin in the dopamine hyperfunction associated with schizophrenia. Schizophr Res 157: 238-243.

Bremer AA, Lustig RH. 2012. Effects of sugar-sweetened beverages on children. Pediatr Ann 41: 26-30.

Burglen F, Marczewski P, Mitchell KJ, van der Linden M, Johnson MK, Danion JM, Salamé P. 2004. Impaired performance in a working memory binding task in patients with schizophrenia. Psychiatry Res 125: $247-255$.

Cameron CM, Carelli RM. 2012. Cocaine abstinence alters nucleus accumbens firing dynamics during goal-directed behaviors for cocaine and sucrose. Eur J Neurosci 35: 940-951.

Colantuoni C, Schwenker J, McCarthy J, Rada P, Ladenheim B, Cadet JL, Schwartz GJ, Moran TH, Hoebel BG. 2001. Excessive sugar intake alters binding to dopamine and $\mu$-opioid receptors in the brain. Neuroreport 12: $3549-3552$

Courtin J, Chaudun F, Rozeske RR, Karalis N, Gonzalez-Campo C, Wurtz H, Abdi A, Baufreton J, Bienvenu TC, Herry C. 2014. Prefrontal parvalbumin interneurons shape neuronal activity to drive fear expression. Nature 505: 92-96.

Curley AA, Lewis DA. 2012. Cortical basket cell dysfunction in schizophrenia. J Physiol 590: 715-724.

DiMeglio DP, Mattes RD. 2000. Liquid versus solid carbohydrate: Effects on food intake and body weight. Int J Obes Relat Metab Disord 24: 794-800.

Dubois L, Farmer A, Girard M, Peterson K. 2007. Regular sugar-sweetened beverage consumption between meals increases risk of overweight among preschool-aged children. J Am Diet Assoc 107: 924-934.

Ennaceur A, Delacour J. 1988. A new one-trial test for neurobiological studies of memory in rats. 1: behavioral data. Behav Brain Res 31: 47-59.

Euston DR, Gruber AJ, McNaughton BL. 2012. The role of medial prefrontal cortex in memory and decision-making. Neuron 76: 1057-1070.

Fowler KS, Saling MM, Conway EL, Semple JM, Louis WJ. 2002. Paired associate performance in the early detection of DAT. J Int Neuropsychol Soc 8: $58-71$.

Frary CD, Johnson RK, Wang MQ. 2004. Children and adolescents' choices of foods and beverages high in added sugars are associated with intakes of key nutrients and food groups. I Adolesc Health 34: 56-63.

Furlong TM, Jayaweera HK, Balleine BW, Corbit LH. 2014. Binge-like consumption of a palatable food accelerates habitual control of behavior and is dependent on activation of the dorsolateral striatum. $J$ Neurosci 34: 5012-5022.

Good MA, Hale G. 2007. The "Swedish" mutation of the amyloid precursor protein (APPswe) dissociates components of object-location memory in aged Tg2576 mice. Behav Neurosci 121: 1180-1191.

Groenewegen HJ. 1988. Organization of the afferent connections of the mediodorsal thalamic nucleus in the rat, related to the mediodorsal-prefrontal topography. Neuroscience 24: 379-431.

Guo X, Park Y, Freedman ND, Sinha R, Hollenbeck AR, Blair A, Chen H. 2014. Sweetened beverages, coffee, and tea and depression risk among older US adults. PLoS One 9: e94715.

Guthrie JF, Morton JF. 2000. Food sources of added sweeteners in the diets of Americans. J Am Diet Assoc 100: 43-51, quiz 49-50.
Haddon JE, Killcross S. 2006. Prefrontal cortex lesions disrupt the contextual control of response conflict. J Neurosci 26: 2933-2940.

Haddon JE, Killcross S. 2007. Contextual control of choice performance: behavioral, neurobiological, and neurochemical influences. Ann N Y Acad Sci 1104: 250-269.

Hajnal A, Norgren R. 2002. Repeated access to sucrose augments dopamine turnover in the nucleus accumbens. Neuroreport 13: 2213-2216.

Howell D. 2013. Fundamental statistics for the behavioral sciences. Cengage Learning.

Hsu TM, Konanur VR, Taing L, Usui R, Kayser BD, Goran MI, Kanoski SE 2015. Effects of sucrose and high fructose corn syrup consumption on spatial memory function and hippocampal neuroinflammation in adolescent rats. Hippocampus 25: 227-239.

Iniguez SD, Warren BL, Parise EM, Alcantara LF, Schuh B, Maffeo ML Manojlovic Z, Bolaños-Guzmán CA. 2009. Nicotine exposure during adolescence induces a depression-like state in adulthood. Neuropsychopharmacology 34: 1609-1624.

Kendig MD, Boakes RA, Rooney KB, Corbit LH. 2013. Chronic restricted access to $10 \%$ sucrose solution in adolescent and young adult rats impairs spatial memory and alters sensitivity to outcome devaluation. Physiol Behav 120: 164-172.

Kesner RP, Ragozzino ME. 2003. The role of the prefrontal cortex in object-place learning: a test of the attribute specificity model. Behav Brain Res 146: $159-165$.

Konradi C, Yang CK, Zimmerman EI, Lohmann KM, Gresch P, Pantazopoulos H, Berretta S, Heckers S. 2011. Hippocampa interneurons are abnormal in schizophrenia. Schizophr Res 131: $165-173$.

Koob GF, Volkow ND. 2010. Neurocircuitry of addiction. Neuropsychopharmacology 35: 217-238.

Lewis DA, Hashimoto T, Volk DW. 2005. Cortical inhibitory neurons and schizophrenia. Nat Rev Neurosci 6: 312-324.

Lewis DA, Curley AA, Glausier JR, Volk DW. 2012. Cortical parvalbumin interneurons and cognitive dysfunction in schizophrenia. Trends Neurosci 35: 57-67.

Lien L, Lien N, Heyerdahl S, Thoresen M, Bjertness E. 2006. Consumption of soft drinks and hyperactivity, mental distress, and conduct problems among adolescents in Oslo, Norway. Am J Public Health 96: 1815-1820.

Lodge DJ, Behrens MM, Grace AA. 2009. A loss of parvalbumin-containing interneurons is associated with diminished oscillatory activity in an animal model of schizophrenia. J Neurosci 29: 2344-2354.

Lustig RH, Schmidt LA, Brindis CD. 2012. Public health: the toxic truth about sugar. Nature 482: 27-29.

MacLeod CM. 1991. Half a century of research on the Stroop effect: An integrative review. Psychol Bull 109: 163-203.

MacLeod CM, MacDonald PA. 2000. Interdimensional interference in the Stroop effect: Uncovering the cognitive and neural anatomy of attention. Trends Cogn Sci 4: 383-391.

Marquis JP, Killcross S, Haddon JE. 2007. Inactivation of the prelimbic, but not infralimbic, prefrontal cortex impairs the contextual control of response conflict in rats. Eur J Neurosci 25: 559-566.

Nelson A, Killcross S. 2006. Amphetamine exposure enhances habit formation. J Neurosci 26: 3805-3812.

Nelson AJ, Hindley EL, Haddon JE, Vann SD, Aggleton JP. 2014. A novel role for the rat retrosplenial cortex in cognitive control. Learn Mem 21: 90-97.

O'Donnell P. 2010. Adolescent maturation of cortical dopamine. Neurotox Res 18: 306-312.

O'Donnell P. 2011. Adolescent onset of cortical disinhibition in schizophrenia: insights from animal models. Schizophr Bull 37: 484-492.

Parsegian A, Glen WB Jr, Lavin A, See RE. 2011. Methamphetamine self-administration produces attentional set-shifting deficits and alters prefrontal cortical neurophysiology in rats. Biol Psychiatry 69: 253-259.

Paus T, Keshavan M, Giedd JN. 2008. Why do many psychiatric disorders emerge during adolescence? Nat Rev Neurosci 9: 947-957.

Paxinos G, Watson C. 2006. The rat brain in stereotaxic coordinates: hard cover edition. Academic Press.

Popkin BM, Nielsen SJ. 2003. The sweetening of the world's diet. Obes Res 11: $1325-1332$.

Porter JN, Olsen AS, Gurnsey K, Dugan BP, Jedema HP, Bradberry CW. 2011. Chronic cocaine self-administration in Rhesus monkeys: impact on associative learning, cognitive control, and working memory. J Neurosci 31: 4926-4934.

Reichelt AC, Good MA, Killcross S. 2013a. Attenuation of acute d-amphetamine-induced disruption of conflict resolution by clozapine, but not alpha-flupenthixol in rats. J Psychopharmacol 27: 1023-1031.

Reichelt AC, Killcross S, Wilkinson LS, Humby T, Good MA. 2013b. Transgenic expression of the FTDP-17 tauV337M mutation in brain dissociates components of executive function in mice. Neurobiol Learn Mem 104: $73-81$. 
Sheludiakova A, Rooney K, Boakes RA. 2012. Metabolic and behavioural effects of sucrose and fructose/glucose drinks in the rat. Eur J Nutr 51: $445-454$.

Shi Z, Taylor AW, Wittert G, Goldney R, Gill TK. 2010. Soft drink consumption and mental health problems among adults in Australia. Public Health Nutr 13: 1073-1079.inter-trial.

Smith RF. 2003. Animal models of periadolescent substance abuse. Neurotoxicol Teratol 25: 291-301.

Stroop JR. 1935. Studies of interference in serial verbal reactions. J Exp Psychol 18: 643.

Stuss DT, Floden D, Alexander MP, Levine B, Katz D. 2001. Stroop performance in focal lesion patients: dissociation of processes and frontal lobe lesion location. Neuropsychologia 39: 771-786.

Thomsen M, Hall FS, Uhl GR, Caine SB. 2009. Dramatically decreased cocaine self-administration in dopamine but not serotonin transporter knock-out mice. J Neurosci 29: 1087-1092.

Timofeeva OA, Levin ED. 2011. Glutamate and nicotinic receptor interactions in working memory: importance for the cognitive impairment of schizophrenia. Neuroscience 195: 21-36.

Tseng KY, O'Donnell P. 2007. Dopamine modulation of prefrontal cortical interneurons changes during adolescence. Cereb Cortex 17: $1235-1240$.

Uylings HB, Groenewegen HJ, Kolb B. 2003. Do rats have a prefrontal cortex? Behav Brain Res 146: 3-17.

Vendruscolo LF, Izidio GS, Takahashi RN, Ramos A. 2008. Chronic methylphenidate treatment during adolescence increases anxiety-related behaviors and ethanol drinking in adult spontaneously hypertensive rats. Behav Pharmacol 19: 21-27.
Vendruscolo LF, Gueye AB, Darnaudéry M, Ahmed SH, Cador M. 2010. Sugar overconsumption during adolescence selectively alters motivation and reward function in adult rats. PLoS One 5: e9296.

Wood SJ, Proffitt T, Mahony K, Smith DJ, Buchanan JA, Brewer W, Stuart GW, Velakoulis D, McGorry PD, Pantelis C. 2002. Visuospatial memory and learning in first-episode schizophreniform psychosis and established schizophrenia: a functional correlate of hippocampal pathology? Psychol Med 32: 429-438.

Yang Q, Zhang Z, Gregg EW, Flanders WD, Merritt R, Hu FB. 2014. Added sugar intake and cardiovascular diseases mortality among US adults. JAMA Intern Med 174: 516-524.

Yawata S, Yamaguchi T, Danjo T, Hikida T, Nakanishi S. 2012. Pathway-specific control of reward learning and its flexibility via selective dopamine receptors in the nucleus accumbens. Proc Natl Acad Sci 109: 12764-12769.

Yu JY, Frank LM. 2014. Hippocampal-cortical interaction in decision making. Neurobiol Learn Mem 117C: $34-41$.

Yu B, He H, Zhang Q, Wu H, Du H, Liu L, Wang C, Shi H, Xia Y, Guo X, et al. 2014. Soft drink consumption is associated with depressive symptoms among adults in China. J Affect Disord 172C: 422-427.

Zamberletti E, Beggiato S, Steardo L Jr, Prini P, Antonelli T, Ferraro L, Rubino T, Parolaro D. 2014. Alterations of prefrontal cortex GABAergic transmission in the complex psychotic-like phenotype induced by adolescent $\delta$-9-tetrahydrocannabinol exposure in rats. Neurobiol Dis 63: $35-47$.

Received December 26, 2014; accepted in revised form February 3, 2015. 


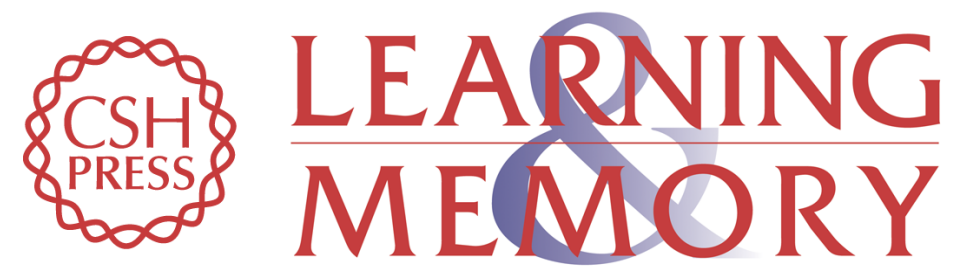

\section{Impact of adolescent sucrose access on cognitive control, recognition memory, and parvalbumin immunoreactivity}

Amy C Reichelt, Simon Killcross, Luke D. Hambly, et al.

Learn. Mem. 2015, 22:

Access the most recent version at doi:10.1101/Im.038000.114

References This article cites 74 articles, 10 of which can be accessed free at: http://learnmem.cshlp.org/content/22/4/215.full.html\#ref-list-1

Creative This article is distributed exclusively by Cold Spring Harbor Laboratory Press for the Commons first 12 months after the full-issue publication date (see

License http://learnmem.cshlp.org/site/misc/terms.xhtml). After 12 months, it is available under a Creative Commons License (Attribution-NonCommercial 4.0 International), as described at http://creativecommons.org/licenses/by-nc/4.0/.

Email Alerting Receive free email alerts when new articles cite this article - sign up in the box at the Service top right corner of the article or click here. 